\title{
Newly created animal model of human postoperative junctional ectopic tachycardia
}

\author{
Jeffrey P. Moak, MD, ${ }^{a}$ Marco A. Mercader, MD, ${ }^{b}$ Dingchao He, MD, ${ }^{c}$ TK. Susheel Kumar, MD, ${ }^{c}$ \\ Gregory Trachiotis, MD, ${ }^{\mathrm{d}}$ Robert McCarter, $\mathrm{ScD},{ }^{\mathrm{e}}$ and Richard A. Jonas, $\mathrm{MD}^{\mathrm{c}}$
}

\begin{abstract}
Objective: Junctional ectopic tachycardia complicates the postoperative recovery from open heart surgery in children. The reported risk factors include younger age, prolonged cardiopulmonary bypass times, and administration of inotropic agents. Junctional ectopic tachycardia occurs early after open heart surgery, in the setting of relative postoperative sinus node dysfunction, and exhibits QRS morphology consistent with an origin from the atrioventricular node or proximal conduction system. Our goal was to develop a reproducible animal model for postoperative junctional ectopic tachycardia.
\end{abstract}

\begin{abstract}
Methods: Eleven pigs, aged 2 to 4 months, underwent open heart surgery after induction of general anesthesia. Electrodes were sewn to the left atrium and right ventricle.

Results: Sinus node dysfunction was created using clamp crushing without or with radiofrequency ablation (successful in 1 of 5 pigs) or sinus node removal (successful in 4 of 4 ). After prolonged cardiopulmonary bypass ( $>120$ minutes) alone and with isoproterenol infusion, no spontaneous junctional ectopic tachycardia developed. Junctional ectopic tachycardia or fascicular tachycardia could be initiated after either slow atrioventricular nodal pathway ablation and/or digoxin administration. Junctional ectopic tachycardia occurred in 8 of 9 pigs (mean ventricular rate, $171 \pm 32 \mathrm{bpm}$ ), and fascicular tachycardia occurred in 9 of 9 pigs (mean ventricular rate, $187 \pm 39 \mathrm{bpm}$ ). His and right bundle recordings confirmed the conduction system origin.

Conclusions: Experimental junctional ectopic tachycardia or fascicular tachycardia can occur in the intraoperative setting of sinus node dysfunction, prolonged cardiopulmonary bypass, and enhanced conduction system automaticity. Conduction system automaticity occurred after either physical injury (ablation or tricuspid valve stretch) or measures to augment the transient inward current of the conduction system (isoproterenol and digoxin). This animal model can serve as the basis to assess new treatments of postoperative junctional ectopic tachycardia. (J Thorac Cardiovasc Surg 2013;146:212-21)
\end{abstract}

Recovery from open heart surgery in infants and children is frequently accompanied by postoperative (PO) arrhythmias. The most clinically deleterious arrhythmia is junctional ectopic tachycardia (JET). ${ }^{1}$ JET frequently results in compromised PO hemodynamics and lengthens the intensive care unit stay. ${ }^{2}$ Enhanced ventricular rates with accompanying atrioventricular (AV) dissociation and myocardial edema result in low cardiac output, promoting the

From the Divisions of Cardiology ${ }^{\mathrm{a}}$ and Cardiovascular Surgery ${ }^{\mathrm{c}}$ and Department of Biostatistics and Informatics, ${ }^{\mathrm{e}}$ Children's National Medical Center, Washington, DC; and Divisions of Cardiology ${ }^{\mathrm{b}}$ and Cardiovascular Surgery, ${ }^{\mathrm{d}}$ George Washington University School of Medicine, Washington, DC.

This study was supported in part by the Research Advisory Counsel, Children's National Medical Center, Washington, DC; Richard B. and Lynne V. Cheney Cardiovascular Institute Research Grant, George Washington University School of Medicine, Washington, DC; and awards UL1RR031988 and P30HD40677 from the National Institutes of Health National Center for Research Resources and National Institutes of Health Intellectual and Developmental Disabilities Research Center.

Disclosures: Authors have nothing to disclose with regard to commercial support. Received for publication April 6, 2012; revisions received Aug 12, 2012; accepted for publication Aug 24, 2012; available ahead of print Sept 28, 2012.

Address for reprints: Jeffrey P. Moak, MD, Division of Cardiology, Children's National Medical Center, 111 Michigan Ave, NW, Washington, DC 20010 (E-mail: jmoak@childrensnational.org).

0022-5223/\$36.00

Copyright (c) 2013 by The American Association for Thoracic Surgery http://dx.doi.org/10.1016/j.jtcvs.2012.08.068 early administration of inotropic agents, which further increase the heart rate. The response of JET to pharmacologic treatment is highly variable and fraught with frequent adverse side effects. ${ }^{3}$

The incidence of PO JET has been reported to vary from $5 \%$ to $15 \%$ of pediatric open heart surgical cases in different case series. ${ }^{4-12}$ Most studies have shown a relation among younger age at surgery, prolonged cardiopulmonary bypass, prolonged aortic crossclamping, and the use of PO inotropic agents at the occurrence of JET. ${ }^{4,5,8-10}$ PO JET tends to occur early after open heart surgery, in the setting of relative sinus node (SN) dysfunction, and exhibits a QRS morphology consistent with an origin from the His bundle (JET) or proximal conduction fascicles (fascicular tachycardia [FT]).

Our goal was to develop a reliable and reproducible animal model similar to human PO JET to use in the development of improved treatment strategies to study in clinical trials.

\section{METHODS \\ Experimental Preparation}

The animal care and use committees of the George Washington School of Medicine and Children's National Medical Center approved the research protocol. All pigs received humane care in compliance with the Guide for the Care and Use of Laboratory Animals. Eleven domestic swine, weighing 


$$
\begin{aligned}
& \text { Abbreviations and Acronyms } \\
& \begin{aligned}
\text { AV } & =\text { atrioventricular } \\
\text { cAMP } & =\text { cyclic adenosine monophosphate } \\
\text { CS } & =\text { conduction system } \\
\text { FT } & =\text { fascicular tachycardia } \\
\text { HV } & =\text { His to ventricle conduction time } \\
\text { JET } & =\text { junctional ectopic tachycardia } \\
\text { PO } & =\text { postoperative } \\
\text { RF } & =\text { radiofrequency } \\
\text { SN } & =\text { sinus node } \\
\text { VA } & =\text { ventriculoatrial }
\end{aligned}
\end{aligned}
$$

$50.5 \pm 13.1 \mathrm{~kg}$ (range, 27-70) and aged 2 to 4.4 months (mean \pm standard deviation, $3.3 \pm 0.8$ ) were used for the present study. Of the 11 pigs, 7 were male and 4 were female. The pigs were brought to the Washington Institute of Surgical Endoscopy laboratory after fasting for 24 hours. Anesthetic induction followed premedication with a combination of $4.4 \mathrm{mg} / \mathrm{kg}$ tiletamine/zolazepam (Telazol) and $2.2 \mathrm{mg} / \mathrm{kg}$ xylazine. The pigs were then intubated and ventilated with room air supplemented with oxygen to maintain normal oxygen saturation using a respirator. Throughout the experiment, oxygen saturation was kept greater than $95 \%$. The body temperature was monitored with a rectal probe and kept at $38^{\circ} \mathrm{C} \pm 1{ }^{\circ} \mathrm{C}$ using a temperature-controlled, heated water blanket.

Anesthesia was maintained with $1 \%$ to $3 \%$ isoflurane, supplemented with midazolam $(0.05-0.1 \mathrm{mg} / \mathrm{kg}$ ) and sufentanil (loading dose of $1 \mu \mathrm{g} /$ $\mathrm{kg} / \mathrm{min}$ and continuous intravenous drip of $1-2 \mu \mathrm{g} / \mathrm{kg} / \mathrm{hr}$ ) throughout the experiment. Normal saline at 100 to $200 \mathrm{~mL} / \mathrm{hr}$ was infused to replace spontaneous fluid and blood losses. The arterial blood gases and electrolytes were monitored using an I-STAT analyzer and EG7 + test cartridges (Abbott Laboratories, Abbott Park, Ill).

Using clean surgical practice, femoral artery and venous catheterization were performed using a combined cut down and Seldinger technique for monitoring arterial blood pressure and gases and central venous pressure, respectively. Two body surface electrocardiographic leads (II and III) were monitored for heart rate and cardiac rhythm assessment. The signals were amplified, digitized, and displayed on a personal computer using an AD Instruments PowerLab 8 data acquisition system with LabChart Pro, version 7.1 (AD Instruments, Colorado Springs, Colo).

After a midline sternotomy incision and with the ribs and lungs retracted, the pericardium was opened and a cradle formed. Two bipolar pairs of pacing wires were sewn to the left atrium to allow for atrial pacing and recording from separate electrodes. One bipolar pair of pacing wires was sewn to the right ventricle for either recording or pacing the ventricle.

The AV node Wenckebach cycle length and antegrade refractory period were determined in the baseline state using a model 5328 programmable stimulator (Medtronic, Inc, Minneapolis, Minn). The AV node Wenckebach cycle length was determined using rapid atrial pacing, and the AV node antegrade refractory period was determined using atrial extrastimulus testing.

\section{Definition of JET and FT}

Although no uniformly accepted definition of PO JET is available, we defined JET for the purposes of the present study as a supraventricular arrhythmia (same QRS morphology as in sinus rhythm) with no preceding $\mathrm{P}$ wave at a rate that exceeded the normal junctional escape rate for age. The pattern of ventriculoatrial (VA) conduction could be 1:1 VA conduction, VA Wenckebach, or dissociated. JET usually exhibited variability in the rate at onset or termination—warm up or cool down—and did not demonstrate sudden onset or termination. The ventricular rate had to be greater than $120 \mathrm{bpm}$. The normal junctional escape rate after creation of SN dysfunction in our studied pigs was usually less than $90 \mathrm{bpm}$. FT was defined similarly, except that the QRS morphology differed from the sinus rhythm QRS morphology and the His to ventricle conduction time (HV) interval was shorter than in sinus rhythm $(\leq 25 \mathrm{~ms})$.

\section{JET Induction}

After completion of the baseline atrial stimulation protocol, cardiopulmonary bypass was instituted to allow induction of JET. Heparin $(200 \mathrm{U} / \mathrm{kg}$ ) was administered for a goal activated clotting time greater than 400 seconds. The superior and inferior vena cava were cannulated using a $20 \mathrm{~F}$ to $32 \mathrm{~F}$, right-angle, wire-reinforced, venous return cannula (Terumo Cardiovascular Systems Corp, Ann Arbor, Mich) after ligating and dividing the azygous vein. The aorta was cannulated using a $10 \mathrm{~F}$ to $14 \mathrm{~F}$ straight Bio-Medicus arterial cannula (Medtronic Inc). Cardiopulmonary bypass was initiated using a Sarns 8000 roller pump (Terumo Cardiovascular Systems Corp) and membrane oxygenator (Terumo CAPIOX oxygenator; Terumo Cardiovascular Systems Corp) and Dideco D733 arterial filter (Sorin Group USA, Inc, Arvada, Colo). The circuit was primed with Plasma-Lyte A to a goal hematocrit greater than $25 \%$. Normothermia was maintained throughout the experiment. Once cardiopulmonary bypass was stable, JET was initiated using the following surgical and pharmacologic maneuvers on a warm beating heart in the following sequence (Figure 1):

Step 1. Crushing the SN with a clamp

Step 2. If crushing the $\mathrm{SN}$ was unsuccessful, radiofrequency (RF) ablation was performed around the superior vena cava-right atrial junction (site of the presumed location of the $\mathrm{SN}$ )

Step 3. If RF ablation was unsuccessful, the snares around the vena cava were tightened, and the right atrium was opened by an incision parallel to the $\mathrm{AV}$ groove, allowing surgical resection of the $\mathrm{SN}$ region

Step 4. Once diminished SN electrical activity was observed (low atrial or junction rhythm), an intravenous infusion of isoproterenol ( 0.01 $0.1 \mu \mathrm{g} / \mathrm{kg} / \mathrm{min}$ ) was started. If JET still did not occur, steps 5 or 6 were performed

Step 5. The right atrium was opened further, with the heart beating. RF ablation lesions were placed along the slow AV nodal pathway to induce accelerated junctional rhythm using an EPT $6 \mathrm{~F}$ ablation catheter and EPT model 1000XP RF generator (Boston Scientific, Natick, Mass). Using an Army-Navy right-angle retractor, the tricuspid valve was stretched during cardiopulmonary bypass for 45 seconds, to its maximum stretch length on 3 occasions. If the rate of accelerated junctional rhythm was less than $120 \mathrm{bpm}$, the rate was accelerated using an isoproterenol infusion $(0.01-0.1 \mathrm{mg} / \mathrm{mg} / \mathrm{min})$

Step 6. Digoxin was administered in small incremental doses until the desired effect was achieved (ie, the development of JET or FT)

\section{Rationale for Experimental Design}

Given the limitations of an acute experiment, we did not have the resources available (intensive care unit and personnel) to allow the pigs to recover from surgery and observe them for 24 to 48 hours for the development of PO JET. As often occurs in humans, PO JET develops shortly after removal of the aortic crossclamp or weaning from cardiopulmonary bypass. Therefore, we designed our protocol to maximize JET occurrence in the immediate operative period. Our personal observations of PO JET reflected the presence of "relative" SN dysfunction in that the sinus rate is slow, given the abnormal hemodynamic state and high degree of catecholamine stimulation frequently present at PO JET occurrence. We, therefore, thought it would be important to induce SN dysfunction in our experimental setting to simulate this element. The accelerated junctional rate responsible for PO JET reflects enhanced automaticity (possibly triggered activity) from this region of the heart and is most likely secondary to mechanical injury (ie, stretch from retraction, sutures) and factors that 


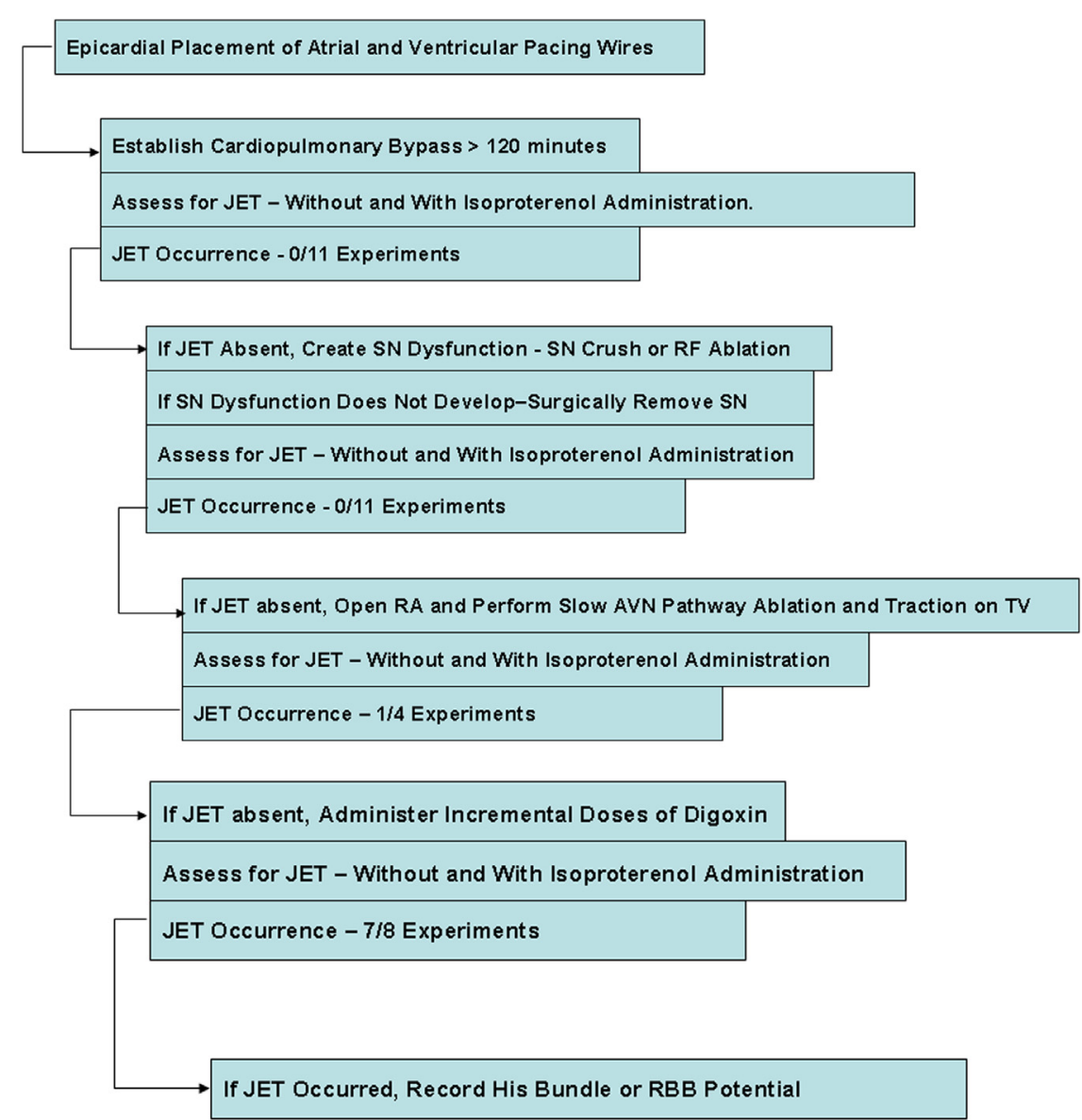

FIGURE 1. Flow diagram of experiment design and frequency of junctional ectopic tachycardia (JET) occurrence. $A V N$, Atrioventricular node; $R A$, right atrium; $R B B$, right bundle branch; $R F$, radiofrequency; $S N$, sinus node; $T V$, tricuspid valve.

might increase the transient inward current that underlies triggered activity. ${ }^{13-16}$ These factors include pharmacologic administration of medications (isoproterenol, dopamine or milrinone) known to increase cyclic adenosine monophosphate (cAMP), myocardial ischemia (prolonged cardiopulmonary bypass, aortic crossclamping, or ventricular tachycardia or fibrillation), and physical trauma to the conduction system (CS). We simulated these elements in our experimental model through slow AV nodal pathway ablation (physical injury and local release of high catecholamines), tricuspid valve stretch (physical injury), and combined pharmacologic administration of isoproterenol and digoxin, agents both known to pharmacologically augment the transient inward current.

\section{His and Right Bundle Branch Recordings}

The His bundle and/or right bundle branch electrograms were recorded using a Daig (Minnetonka, Minn), 4F quadrapolar catheter and an AD Instruments PowerLab 8 data acquisition system with LabChart Pro 7.1 (AD Instruments) under direct visualization of the $\mathrm{AV}$ node and right bundle branch.

\section{Statistical Analysis}

Data are reported as the mean \pm standard deviation.

\section{RESULTS}

\section{Baseline AV Node Function}

After placing the temporary pacing leads on the left atrium and right ventricle, the AV node Wenckebach cycle length and antegrade AV node effective refractory periods were determined in the control state. The antegrade Wenckebach cycle length measured $254 \pm 52 \mathrm{~ms}$, and the antegrade AV node effective refractory period was determined at an average paced cycle length of $456 \pm 65 \mathrm{~ms}$ and measured $257 \pm 59 \mathrm{~ms}$. No atrial arrhythmias were induced during atrial stimulation.

\section{Induction of SN Dysfunction}

Clamp crushing without or with RF ablation of the SN region was successful in 1 of 5 attempts (Table 1). Surgical removal of the SN was success in 4 of 4 attempts (Figure 2). After surgical removal of the SN region, the cardiac rhythm transitioned to sinus bradycardia, a low right atrial rhythm, junctional rhythm, or fascicular rhythm. When junctional rhythm occurred after SN removal and with minimal catecholamine infusion, the ventricular rate was less than $90 \mathrm{bpm}$.

\section{Junctional Ectopic Tachycardia}

JET occurred after RF ablation of the slow AV nodal pathway alone in 1 pig, slow pathway ablation and digoxin administration in 3 pigs, and after digoxin administration 
BP

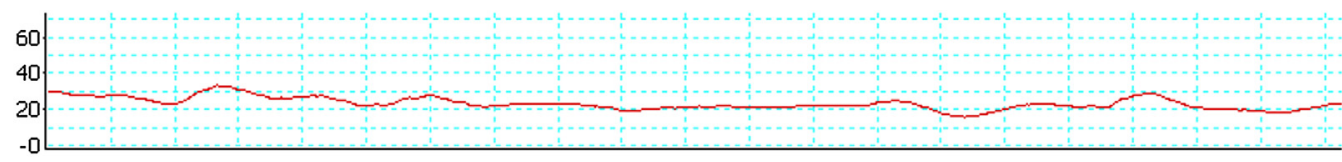

ECG 1

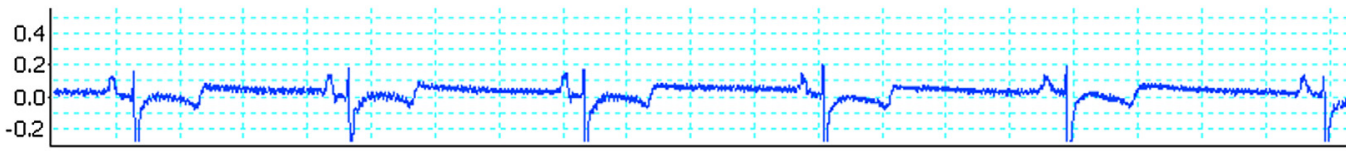

ECG 2

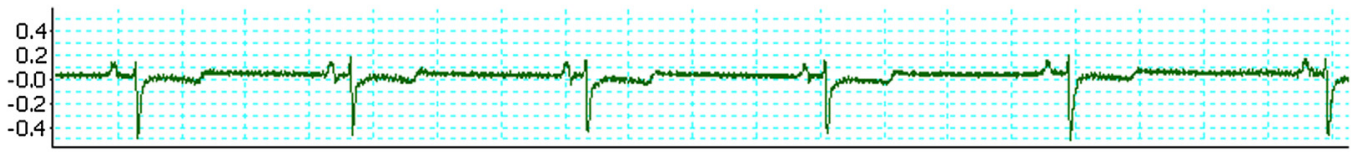

Arial ELG

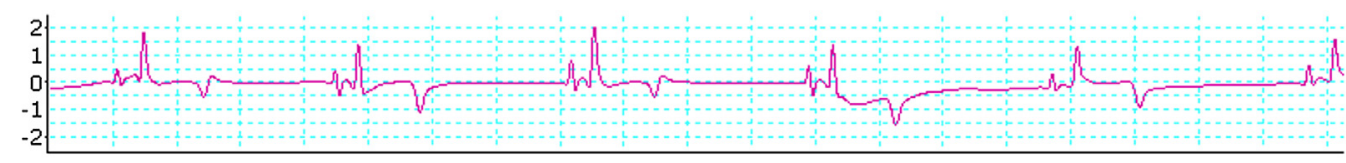

Vent ELG

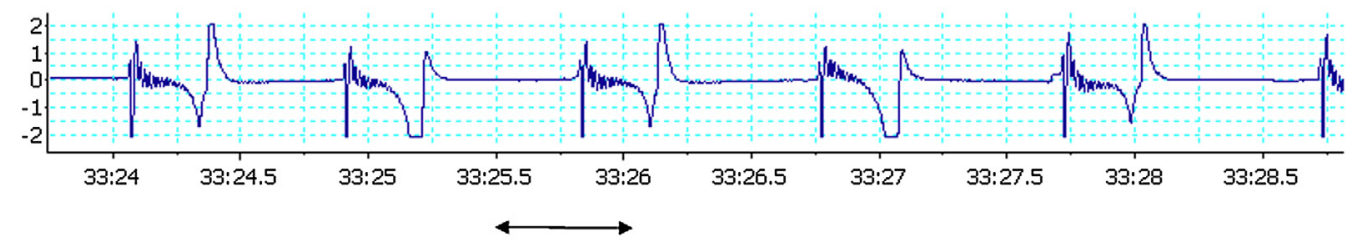

A

$500 \mathrm{msec}$

BP

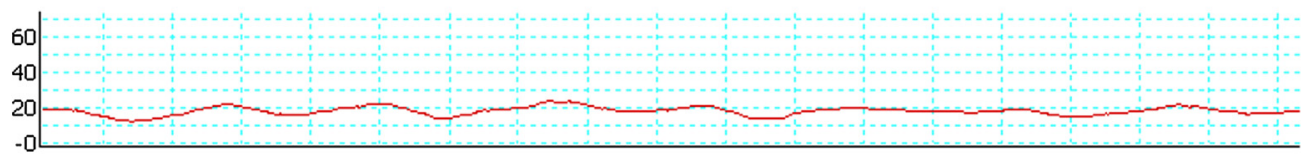

ECG 1

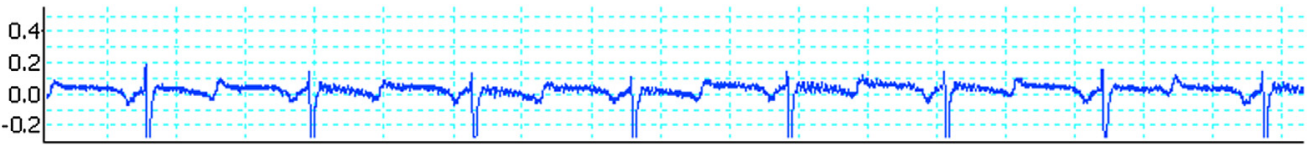

ECG 2

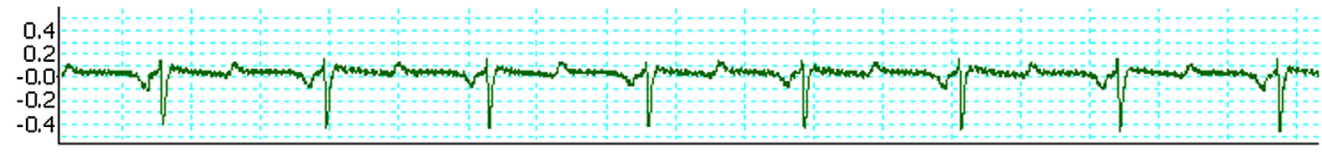

Atrial ELG

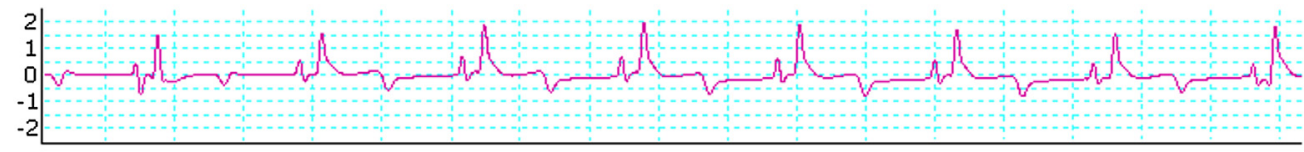

Vent ELG

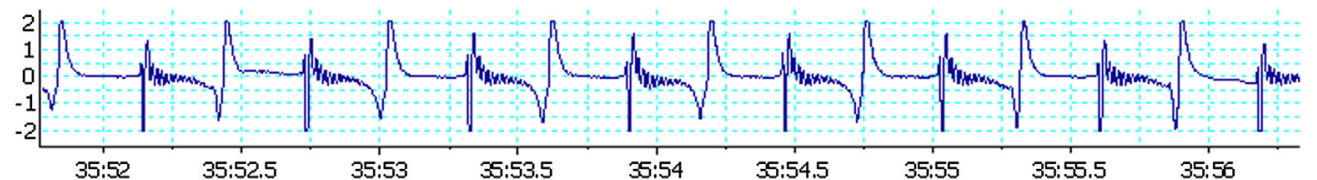

B

$500 \mathrm{msec}$

FIGURE 2. Sinus node $(S N)$ dysfunction after removal of SN region and right atrial $(R A)$ appendage during cardiopulmonary bypass. After removal of SN region and RA appendage, 1 of 4 cardiac rhythms ensued: A, sinus bradycardia to a cycle length of $950 \mathrm{~ms}$; B, low RA rhythm at a cycle length of $625 \mathrm{~ms}$; $\mathrm{C}$, junctional rhythm with 1:1 ventriculoatrial (VA) conduction at a cycle length of $880 \mathrm{~ms}$. His bundle electrogram [arrow] precedes local ventricular electrogram by $30 \mathrm{~ms}$; and D, fascicular rhythm at a cycle length of $920 \mathrm{~ms}$ with 1:1 VA conduction. Note change in QRS morphology compared with that in panel C. The format of the tracings is blood pressure $(B P)$, electrocardiogram 1 ( $E C G$ 1, lead 2), electrocardiogram 2 (ECG 2, lead 3), left atrial electrogram (Atrial $E L G$ ), and right ventricular electrogram (Vent $E L G$ ) or His bundle recording. Time axis is denoted at bottom of tracing. 
BP

ECG1

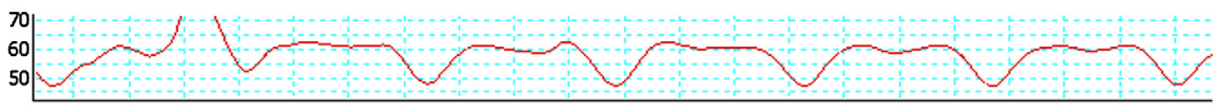

ECG 2

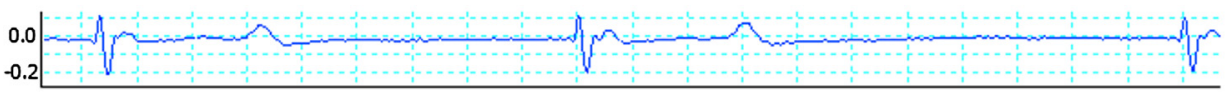

ECG 2

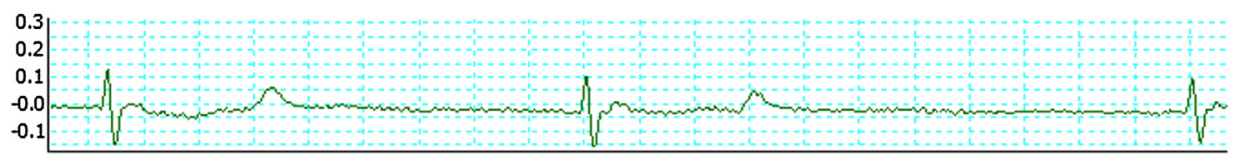

Atrial ELG

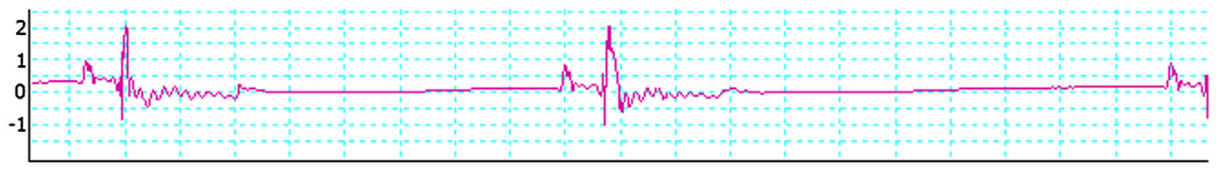

His ELG

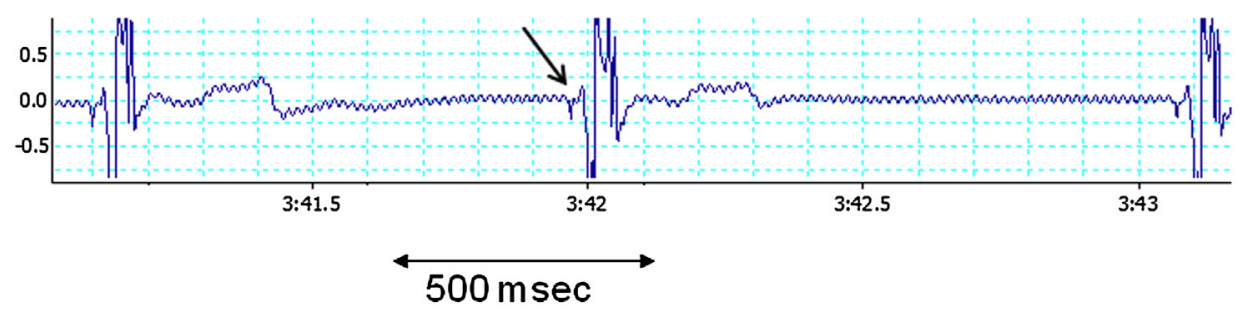

BP

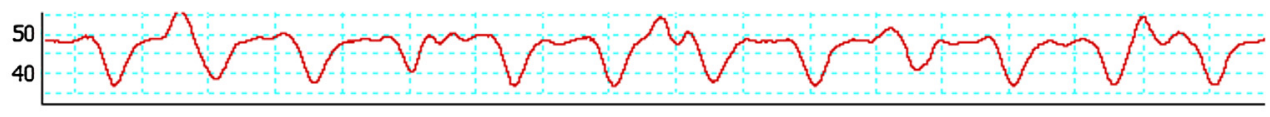

ECG1

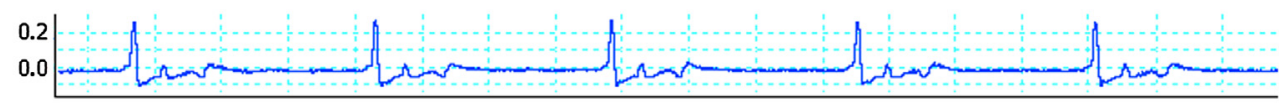

ECG 2

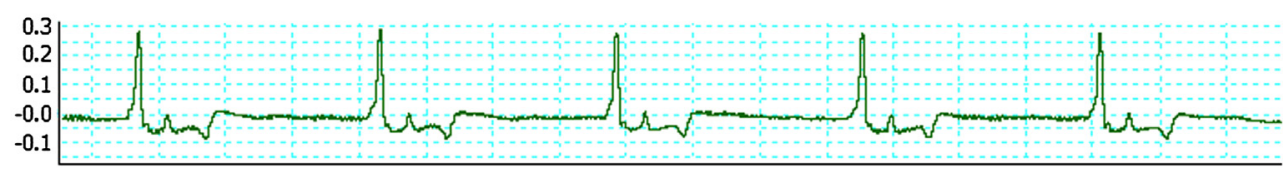

Atrial ELG

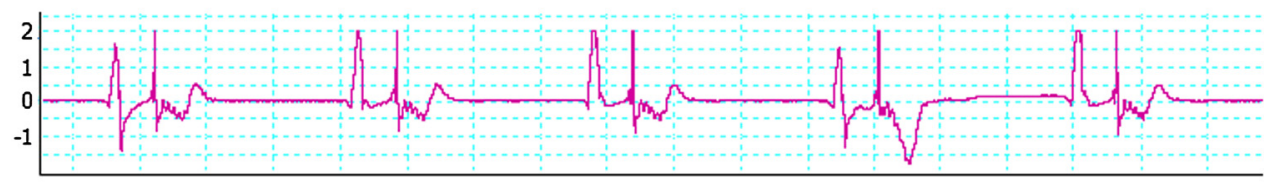

\section{Vent ELG}

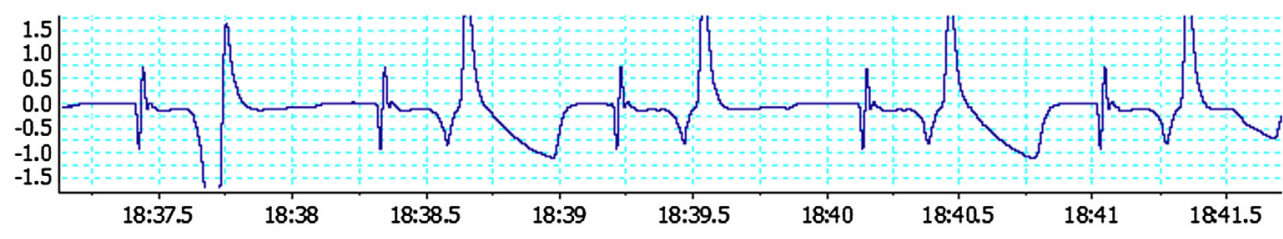

D

FIGURE 2. (continued).

alone in 4 pigs (Table 1). The mean rate of JET was $171 \pm 32$ bpm. Figure 3, $A$, demonstrates an example of JET at a rate of $175 \mathrm{bpm}$ with VA dissociation and a normal His-ventricle conduction time (HV) interval. To prove that the QRS morphology was unchanged during JET, rapid atrial pacing was performed at $180 \mathrm{bpm}$. Note the identical QRS morphology to JET during rapid atrial pacing (Figure 3, $B$ ). JET occurred in 8 pigs, and 2 pigs died of malignant hyperkalemia. Ventricular tachycardia with hypotension or ventricular fibrillation occurred in 8 of 8 pigs with JET during either cardiopulmonary bypass $(n=6)$ or before bypass $(n=2)$. JET duration averaged $7 \pm 2$ minutes. 
TABLE 1. Experimental interventions performed and occurrence of JET or FT

\begin{tabular}{|c|c|c|c|c|c|c|c|c|}
\hline $\begin{array}{c}\text { Experiment } \\
\text { no. } \\
\end{array}$ & SN & $\begin{array}{c}\text { Slow AV nodal } \\
\text { pathway RFA }\end{array}$ & $\begin{array}{c}\text { Digoxin } \\
(\text { dose }-\mu \mathrm{g} / \mathrm{kg})\end{array}$ & $\begin{array}{c}\text { VT with hypotension } \\
\text { or VF during } \\
\text { experiment } \\
\end{array}$ & $\begin{array}{c}\text { JET rate } \\
(\text { bpm })\end{array}$ & $\begin{array}{c}\text { FT rate } \\
(\text { bpm })\end{array}$ & $\begin{array}{c}\text { JET duration } \\
(\mathrm{min})\end{array}$ & $\begin{array}{c}\begin{array}{c}\text { FT duration } \\
(\mathrm{min})\end{array} \\
\end{array}$ \\
\hline 1 & Crush & $\mathrm{P}$ & NP & $\mathrm{O}$ & 154 & 179 & 2 & 0.7 \\
\hline 2 & Crush and RFA & $\mathrm{P}$ & 92 & $\mathrm{O}$ & 182 & 126 & 5.6 & 1.8 \\
\hline 3 & SN removal & $\mathrm{P}$ & 50 & $\mathrm{O}$ & 116 & 169 & 2.8 & 1.0 \\
\hline 4 & SN removal & $\mathrm{P}$ & 100 & $\mathrm{O}$ & 171 & 205 & 0.15 & 1.6 \\
\hline 5 & Hyperkalemia & NP & NP & DNO & DNO & DNO & - & - \\
\hline 6 & Hyperkalemia & NP & NP & DNO & DNO & DNO & - & - \\
\hline 7 & Crush & NP & 163 & $\mathrm{O}$ & 188 & 254 & 4.0 & 4.0 \\
\hline 8 & Crush and RFA & NP & 189 & DNO & DNO & 189 & - & 0.2 \\
\hline 9 & Crush & NP & 74 & $\mathrm{O}$ & 174 & 203 & 5.0 & 53 \\
\hline 10 & SN removal & NP & 74 & $\mathrm{O}$ & 170 & 180 & 10 & 40 \\
\hline 11 & SN removal & NP & 24 & $\mathrm{O}$ & 181 & 172 & 25 & 0.5 \\
\hline
\end{tabular}

$J E T$, Junctional ectopic tachycardia; $F T$, fascicular tachycardia; $S N$, sinus node; $A V$, atrioventricular; $R F A$, radiofrequency ablation; $V T$, ventricular tachycardia; $V F$, ventricular fibrillation; $P$, performed; $N P$, not performed during experiment; $O$, occurred; $D N O$, did not occur during the experiment.

\section{Fascicular Tachycardia}

FT was identified as a rapid rhythm with QRS morphology different from sinus rhythm or JET and a shorter Hisventricle conduction time (HV) interval (Figure 4). The mean FT rate was $187 \pm 39 \mathrm{bpm}$. FT occurred in 9 pigs. Ventricular tachycardia with hypotension or ventricular fibrillation occurred in 8 of 9 pigs with FT during either cardiopulmonary bypass $(n=6)$ or before bypass $(n=2)$. FT duration averaged $11 \pm 19$ minutes.

\section{DISCUSSION}

Our research study showed the feasibility of creating a reliable and reproducible animal model of human PO JET. Previously, no animal model of PO JET has been available to study to understand the conditions for its development and to use in therapeutic trials. Experimental PO JET or FT occurred in the experimental setting of relative SN dysfunction, and enhanced automaticity of the His-Purkinje CS. CS automaticity occurred after either physical injury to the AV CS (slow AV nodal pathway ablation and tricuspid valve stretch) or pharmacologic interventions that enhance automaticity from the His CS (increased transient inward current secondary to isoproterenol and digoxin administration). ${ }^{13-16}$ Using our experimental design, JET and FT occurred in 8 and 9 pigs, respectively, in a total of 11 animal studies.

JET and FT are common tachyarrhythmias that occur in the early PO period after surgical repair of congenital heart disease, with a reported incidence of 5\% to $15 \%{ }^{4-12}$ JET and FT are usually self-limited arrhythmias lasting 2 to 5 days postoperatively; however, they can result in serious hemodynamic deterioration and can compromise PO recovery. Low cardiac output can necessitate mechanical circulatory support (extracorporeal membrane oxygenation). ${ }^{17,18}$ Hemodynamic instability is caused by the loss of AV synchrony and rapid ventricular rates. JET can be associated with $\mathrm{AV}$ dissociation or a short VA interval when 1:1 retrograde VA conduction occurs. The short VA interval results in atrial contraction during periods of $\mathrm{AV}$ valve closure, simulating pacemaker syndrome. Pharmacologic therapy has limited success and can result in significant morbidity ${ }^{3}$; hence, our interest in understanding the mechanisms for PO JET and developing an animal model to test new treatment options.

Retrospective clinical reviews have attempted to identify specific patient and operative risk factors that can predict the occurrence of JET. ${ }^{4,5-8-10}$ Although predictive outcome variables differed among these studies, certain clinical factors seem common: younger age at surgery, longer cardiopulmonary bypass time, longer aortic crossclamp time, greater level of PO creatine kinase-MB or troponin (reflecting the extent of surgical trauma or myocardial ischemia), high inotrope requirement, and congenital heart disease/surgical intervention type. Many investigators have found an association between congenital heart defects requiring ventricular septal defect closure, such as tetralogy of Fallot and atrioventricular septal defect, with a greater probability of PO JET occurrence. ${ }^{4,10}$ Others studies have demonstrated the frequent occurrence of JET after surgical correction of congenital heart defects not associated with ventricular septal defect closure (ie, arterial switch operation for d-transposition of the great arteries or total cavopulmonary connection for a single ventricle). ${ }^{12}$ Therefore, JET can develop after surgical intervention with minimal or no operative manipulation near or around the AV node and His CS, suggesting that physical trauma to the $\mathrm{AV}$ node and proximal His region might not be a prerequisite for JET occurrence.

\section{PO JET or FT Development}

We were pleased to see the occurrence of periods of JET and FT in the course of our animal experiments. In our 


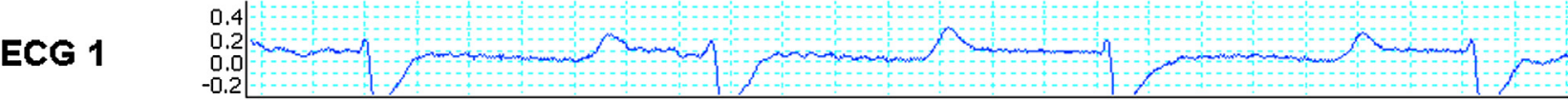

ECG 2
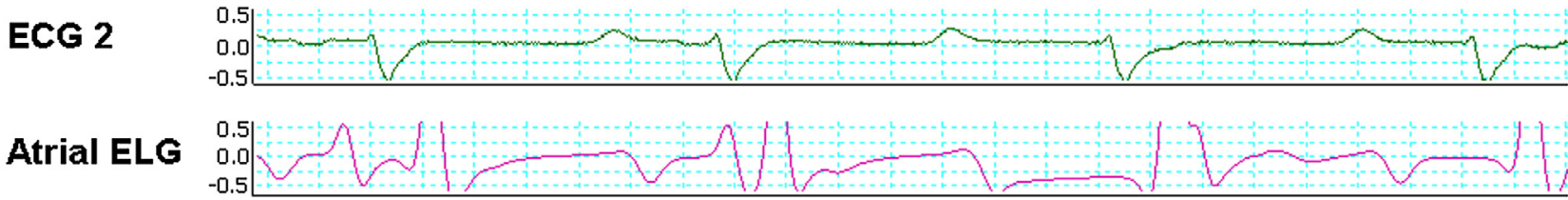

His ELG

A

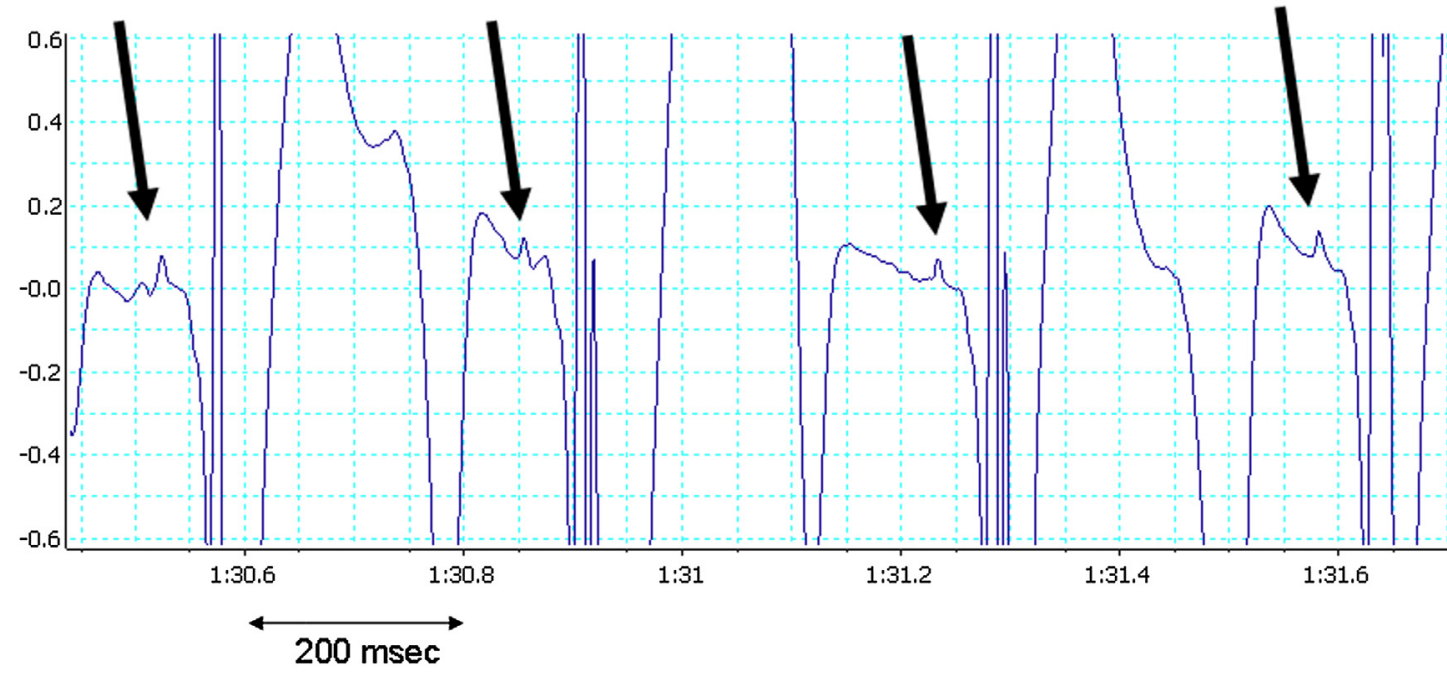

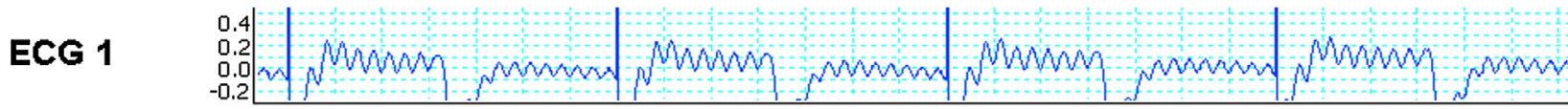

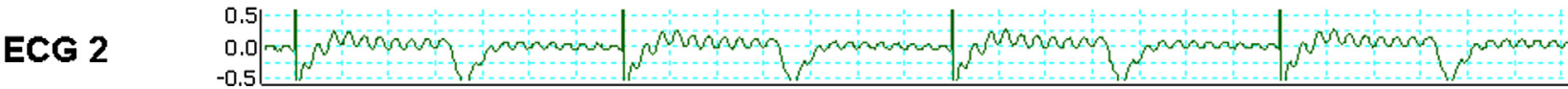

Atrial ELG

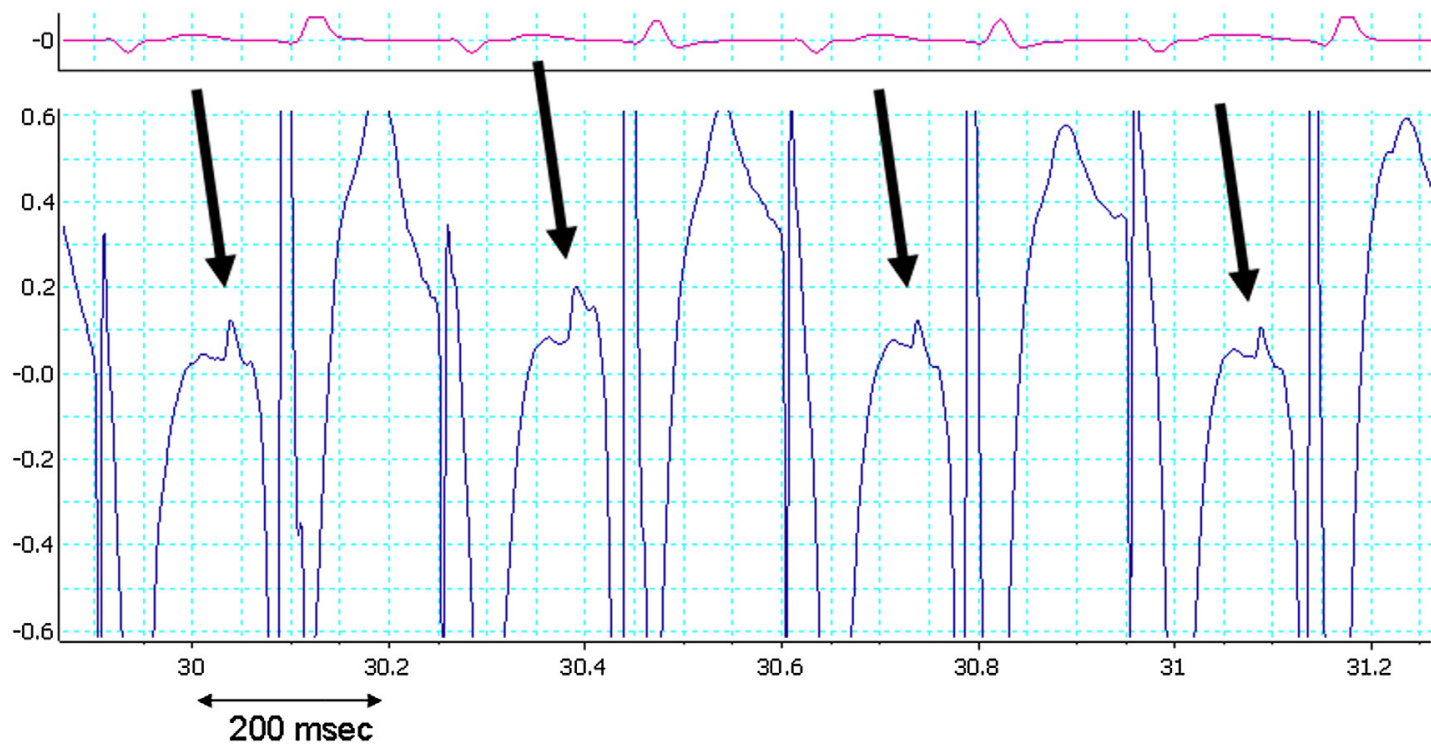

B

His ELG

FIGURE 3. Junctional ectopic tachycardia (JET). A, JET (rate, $175 \mathrm{bpm}$ ) with ventriculoatrial (VA) dissociation at a cycle length of $345 \mathrm{~ms}$. Arrows denote His bundle electrogram $(E L G)$. Local His to ventricular electrogram measured $30 \mathrm{~ms}$. B, Rapid atrial pacing (rate, $180 \mathrm{bpm}$ ) at a cycle length of $333 \mathrm{~ms}$, reestablished atrial paced rhythm with QRS morphology during atrial pacing identical to JET. His-ventricular electrogram interval during atrial pacing was identical to that of JET, proving its origin from atrioventricular $(A V)$ junction. $E C G$, Electrocardiogram. 
BP

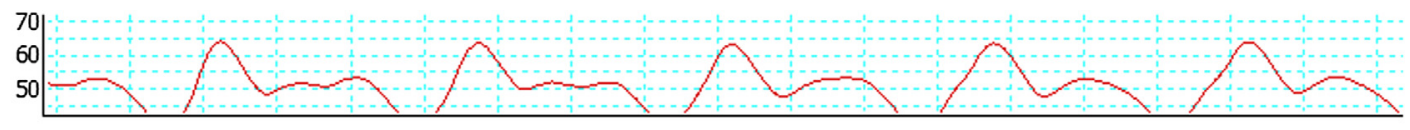

ECG1

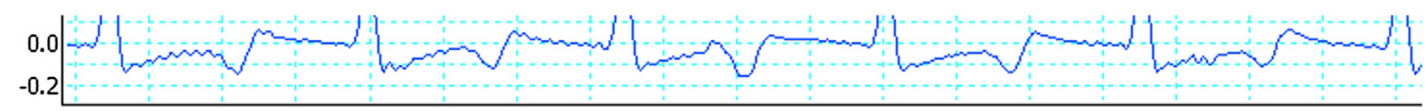

ECG 2

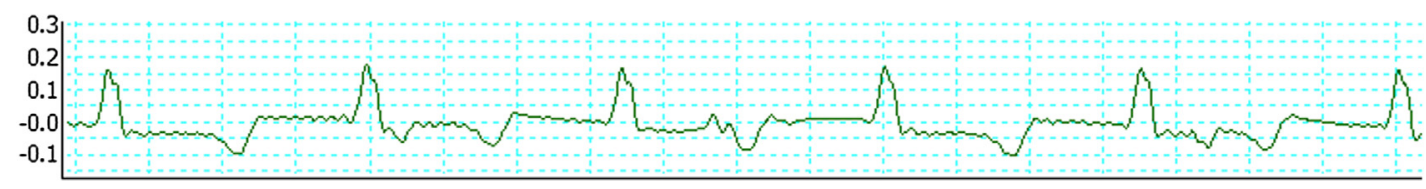

\section{Atrial ELG}

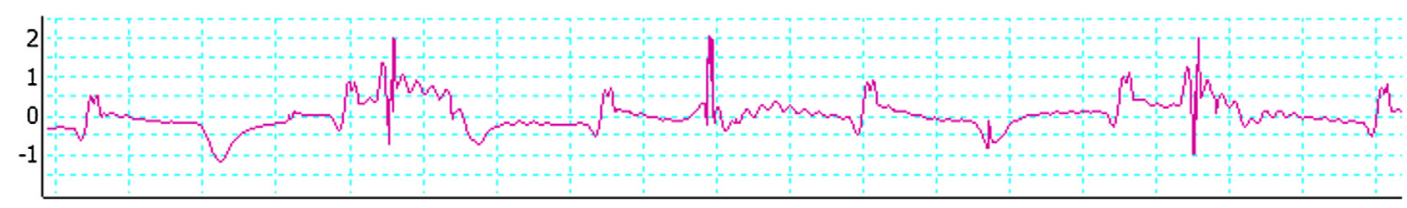

Vent ELG

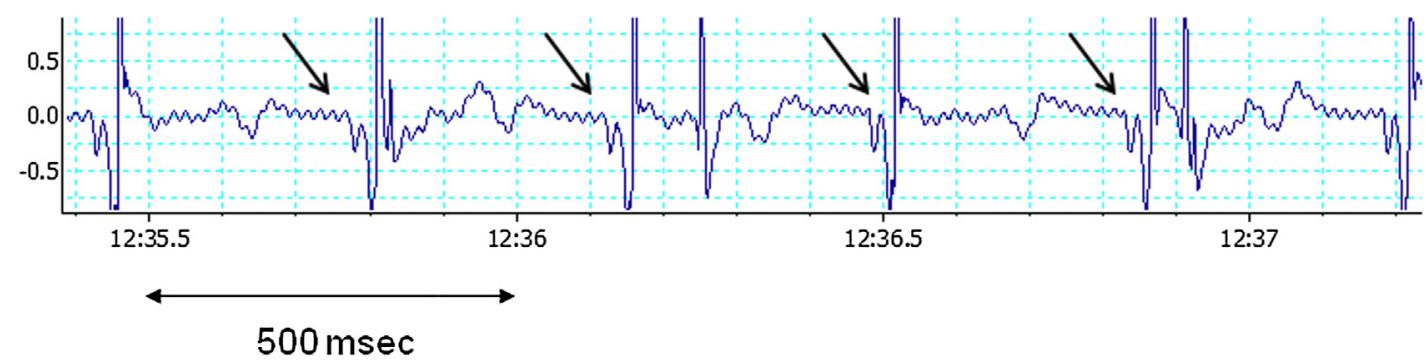

FIGURE 4. Fascicular tachycardia $(F T)$ at a cycle length of $350 \mathrm{~ms}$ with ventriculoatrial (VA) dissociation (ventricular rate, $170 \mathrm{bpm}$ ). Note QRS morphology is similar to that in Figure 2, D. His to local ventricular electrogram measured $20 \mathrm{~ms}$. His bundle electrogram identified by arrows. BP, Blood pressure; $E C G$, electrocardiogram; Atrial $E L G$, left atrial electrogram; Vent $E L G$, right ventricular electrogram.

experience at Children's National Medical Center, we have observed that FT was labeled by the cardiac intensive care unit team as JET in $10 \%$ to $20 \%$ of the cases of PO human "JET" (unpublished data). The exact site of origin of "JET" is not known. We suspect that variability can occur in whether "JET" originates from the proximal or distal part of the AV node-His bundle CS axis. If the JET originates from the more distal part of the His bundle around the site of bifurcation of the His bundle, it will appear as FT. We have observed human cases of JET during which the QRS morphology at times was consistent with JET and at other times was consistent with FT. Therefore, our animal model of JET reproduced our clinical observation of human PO "JET." Successful ablation of JET has been reported in various locations, including the posterior septum (presumed origin from the slow AV nodal pathway), midseptum (presumed origin from the proximal peri-AV node), and the anterior triangle of Koch (presumed origin from the proximal His bundle). ${ }^{19}$

Available clinical evidence suggests that JET and FT are automatic arrhythmias with an underlying cellular electrophysiology of triggered activity or abnormal automaticity. ${ }^{13-16}$ JET tends to occur in 1 of the following clinical settings: (1) physical injury to the AV CS, such as occurs during surgical manipulation of the atrial or ventricular septum, tricuspid valve retraction or suture placement in or around the $\mathrm{AV}$ node-His bundle region; (2) cardiac ischemia (prolonged cardiopulmonary bypass with aortic crossclamping); or (3) after administration of pharmacologic interventions that enhance abnormal automaticity or triggered activity from the proximal His CS (inotropic agents). We sought to determine how each of these operative factors might facilitate PO JET and thus incorporated them into our animal model of PO JET.

\section{Physical Injury to the CS}

Enhanced junctional automaticity can appear after slow $\mathrm{AV}$ nodal pathway ablation when performed using $\mathrm{RF}$ ablation, such as was observed in our study. Several investigators have reported persistent junctional rhythm after RF ablation of the slow $\mathrm{AV}$ nodal pathway to be a highly predictive measure of successful ablation, reflecting more intense injury from the RF ablation on the peri-AV nodal tissue. ${ }^{20-22}$ Junctional tachycardia can last seconds to more than 1 hour after RF ablation. ${ }^{18}$ Although it is unclear, PO JET might have its origin from the slow AV nodal pathway; thus, to facilitate PO JET, we attempted physical injury (RF ablation and tricuspid valve stretch) to this heart region. 


\section{Increased Automaticity and Triggered Activity}

Irons and Orgain ${ }^{23}$ found nonparoxysmal junctional tachycardia was 1 of the more common arrhythmias associated with digitalis toxicity. At the cellular level, digitalis inhibits the sodium-potassium adenosine triphosphate pump and can lead to intracellular calcium overload, delayed after depolarizations, and triggered activity. ${ }^{16}$

Our intent in administering digoxin was to provide an additional stimulus to increase spontaneous automaticity from the $\mathrm{AV}$ junctional region, after the heart had been preconditioned from the effects of prolonged cardiopulmonary bypass. Triggered activity is thought to be the predominant cellular electrophysiologic mechanism underling JET. Digoxin is known to facilitate triggered activity. We, therefore, used digoxin, not to create toxicity, but to help facilitate the occurrence of this arrhythmia. The intravenous administration of PO inotropes has been shown to be 1 of the best predictors of PO JET occurrence. How do these agents bring about PO JET? Milrinone and similar agents increase intracellular cAMP, which is known to also facilitate triggered activity. We therefore used 2 pharmacologic agents (isoproterenol and digoxin), both of which are known to facilitate triggered activity, the underlying cellular mechanism for PO JET.

Wieland and Marchlinski ${ }^{24}$ noted the gradual slowing of digoxin-toxic FT to Fab antibody fragments, change in morphology from multiform to monomorphic tachycardia, and a flat resetting response of the first return cycle of tachycardia to spontaneous premature ventricular beats. All these electrophysiologic features are consistent with an automatic mechanism, such as triggered activity, rather than a reentrant circuit being the underlying mechanism for JET or FT.

Although the dosage of digoxin used in our experimental setting might seem high, the drug was administered with the pigs on cardiopulmonary bypass, which is known to markedly increase the volume of distribution of pharmacologic agents. Digoxin was chosen to help facilitate enhanced junctional automaticity combined with an inotropic agent known to increase intracellular cAMP and triggered activity. Trough digoxin serum levels were not available.

\section{Induction of SN Dysfunction}

A "relative" sinus bradycardia frequently accompanies the development of JET. Therefore, we wished to include this element in our animal model. We had difficulty in our experiments creating reproducible $\mathrm{SN}$ dysfunction using a simple clamp crush technique alone. Sinus bradycardia and pauses were more successfully created by removing a large section of the right atrial free wall. Betts and colleagues, ${ }^{25}$ using 3-dimensional mapping of the right atrium in a swine animal model, showed the complexity of right atrial activation during sinus rhythm. They demonstrated 2 patterns of earliest right atrial endocardial activation: (1) laterally at a position consistent with the terminal crest and (2) superiorly at the junction of the superior caval vein and right atrial appendage. Small changes in sinus cycle length resulted in 5- to $55-\mathrm{mm}$ shifts in the site of pacemaker origin that were idiocentric to each animal and occurred in an unpredictable pattern. ${ }^{26}$ Other investigators using a canine animal model have reported using a diverse range of techniques to induce $\mathrm{SN}$ dysfunction. ${ }^{27,28}$ Crush of the SN was successful only when done at a macroscopic level (ie, a large amount of right atrial tissue, 2 to $3 \mathrm{~cm}$ of sulcus terminalis tissue distal to the $\mathrm{SN}$, was injured or removed). In the absence of catecholamine infusion, the junctional rhythm was always less than $90 \mathrm{bpm}$, significantly slower than the rate of JET or FT we observed in our animal model.

Tan and colleagues ${ }^{29}$ attempted to develop an animal model of ectopic atrial tachycardia (such as might be seen in PO cardiovascular surgery patients). The purpose of their study was to determine whether the thoracic veins (pulmonary veins) might act as ectopic pacemakers. They performed right and left stellate ganglia stimulation before and after SN crushing. Stellate ganglia stimulation triggered significant elevations of norepinephrine levels, sinus tachycardia, and atrial tachycardia in 2 of 8 dogs. SN crushing resulted in a slow junctional rhythm (rate, $51 \mathrm{bpm})$. Subsequent stellate ganglia stimulation induced 20 episodes of ectopic beats in 7 dogs and 7 episodes of pulmonary vein tachycardia in 3 dogs (cycle length, 273; duration, 16 s). Similar to our model, decreased SN function facilitated the occurrence of rapid atrial tachycardia (faster than what the underlying sinus rate would have been). The suppression of SN activity was important in facilitating the expression of these latent arrhythmias, just as we observed in our JET animal model.

Both models demonstrate the importance of pharmacologic stimulation of the transient inward current in manifesting clinical tachyarrhythmias, through either increased intracellular cAMP (norepinephrine and isoproterenol) or intracellular calcium overload (sodium-potassium adenosine triphosphatase inhibition [digoxin]). The development of pulmonary vein tachycardia required similar experimental manipulations, including suppression of SN function and increased intracellular cAMP to augment triggered activity in the latent pacemaker cells in the pulmonary veins. In our model of PO JET, isoproterenol and digoxin were used in concert to augment triggered activity in the latent pacemaker cells in the slow AV nodal pathway (JET) or His bundle (FT).

\section{Study Limitations}

Because of a limited total number of animal experiments, we could not test, in statistical detail, the primary and sequential effect of our 3 study interventions (slow AV nodal pathway ablation, prolonged cardiopulmonary bypass, and digoxin administration). 


\section{CONCLUSIONS}

Our study has shown the feasibility of creating a reliable and reproducible animal model of human PO JET. Experimental PO JET or FT occurred in the setting of "relative" SN dysfunction and enhanced automaticity of the HisPurkinje CS. CS automaticity occurred after either physical injury to the AV CS or pharmacologic interventions that enhance automaticity from the His CS. We hope to use this animal model of human PO JET to develop improved treatment strategies. If the treatment strategies are successful, we then hope to apply them to the development of trials and studies in humans.

\section{References}

1. Grosse-Wortmann L, Kreitz S, Grabitz RG, Vazquez-Jimenez JF, Messmer BJ, von Bernuth $\mathrm{G}$, et al. Prevalence of and risk factors for perioperative arrhythmias in neonates and children after cardiopulmonary bypass; continuous Holter monitoring before and for three days after surgery. J Cardiovasc Surg. 2010;5:85-92.

2. Dodge-Khatami A, Miller OI, Anderson RH, Gil-Jaurena JM, Goldman AP, de Leval MR. Impact of junctional ectopic tachycardia on postoperative morbidity following repair of congenital heart defects. Eur J Cardiovasc Surg. 2002;21: 255-9.

3. Saul JP, Scott WA, Brown S, Marantz P, Acevedo V, Etheridge SP, et al., Intravenous Amiodarone Pediatric Investigators. Intravenous amiodarone for incessant tachyarrhythmias in children: a randomized, double-blind, antiarrhythmic drug trial. Circulation. 2005;29:3470-7.

4. Mildh L, Hiippala A, Rautiainen P, Pettila V, Sairanen H, Happonen JM. Junctional ectopic tachycardia after surgery for congenital heart disease: incidence, risk factors and outcome. Eur J Cardiothorac Surg. 2011;39:75-80.

5. Andreasen JB, Johnsen SP, Ravn HB. Junctional ectopic tachycardia after surgery for congenital heart disease in children. Intensive Care Med. 2008;34: 895-902.

6. Delaney JW, Moltedo JM, Dziura JD, Kop GS, Synder CS. Early postoperative arrhythmias after pediatric cardiac surgery. J Thoracic Cardiovasc Surg. 2006; 131:1296-301.

7. Rekawek J, Kansy A, Miszczak-Knecht M, Manowska M, Bieganowska K, Brzezinska-Paszke M, et al. Risk factors for cardiac arrhythmias in children with congenital heart disease after surgical intervention in the early postoperative period. J Thoracic Cardiovasc Surg. 2007;133:900-4.

8. Batra AS, Chun DS, Johnson TR, Maldonado EM, Kashyap BA, Maiers J, et al. A prospective analysis of the incidence and risk factors associated with junctional ectopic tachycardia following surgery for congenital heart disease. Pediatr Cardiol. 2006;27:51-5.

9. Hoffman TM, Bush DM, Wernovsky G, Cohen MI, Wieand TS, Gaynor JW, et al. Postoperative junctional ectopic tachycardia in children: incidence, risk factors, and treatment. Ann Thorac Surg. 2002;74:1607-11.

10. Dodge-Khatami A, Miller OI, Anderson RH, Goldman AP, Gil-Jaurena JM, Elliot MJ, et al. Surgical substrates of postoperative junctional ectopic tachycardia in congenital heart defects. J Thoracic Cardiovasc Surg. 2002;123:624-30.

11. Pfammatter JP, Wagner B, Berdat P, Bachmann DCG, Pavlovic M, Pfenninger J, et al. Procedural factors associated with early postoperative arrhythmias after repair of congenital heart defects. J Thoracic Cardiovasc Surg. 2002;123:258-62.
12. Cecchin F, Johnsrude CL, Perry JC, Friedman RA. The effect of age and surgical technique on symptomatic arrhythmias after the Fontan procedure. Am J Cardiol. 1995;76:386-91.

13. Santinelli V, De Paola M, Smimmo D, Turco P, Condorelli M. Junctional ectopic tachycardia in adults: role of triggered activity. Chest. 1987;92:188-9.

14. Rosen MR, Fisch C, Hoffman BF, Danilo P Jr, Lovelace DE, Knoebel SB. Can accelerated atrioventricular junctional escape rhythms be explained by delayed after depolarizations? Am J Cardiol. 1980;45:1272-84.

15. Liu CF, Ip JE, Lin AC, Lerman BB. Mechanistic heterogeneity of junctional ectopic tachycardia in adults. Pacing Clin Electrophysiol. Sept 28, 2011 [Epub ahead of print].

16. Moak JP, Rosen MR. Induction and termination of triggered activity by pacing in isolated canine Purkinje fibers. Circulation. 1984;69:149-62.

17. Dyamenahalli U, Tuzcu V, Fontenot E, Pagaiannis J, Jaquiss R, Bhutta A, et al Extracorporeal membrane oxygenation support for intractable primary arrhythmias and complete congenital heart block in newborns and infants: short-term and medium-term outcomes. Pediatr Crit Care Med. April 21, 2011 [Epub ahead of print].

18. Darst JR, Kaufman J. Case report: an infant with congenital junctional ectopic tachycardia requiring extracorporeal mechanical oxygenation. Curr Opin Pediatr. 2007;19:597-600.

19. Hamdan MH, Badhwar N, Scheinman MM. Role of invasive electrophysiologic testing in the evaluation and management of adult patients with focal junctional tachycardia. Card Electrophysiol Rev. 2002;6:431-5.

20. Poret P, Leclercq C, Gras D, Mansour H, Fauchier L, Daubert C, et al. Junctional rhythm during slow pathway radiofrequency ablation in patients with atrioventricular nodal reentrant tachycardia: beat-to-beat analysis and its prognostic value in relation to electrophysiologic and anatomic parameters. J Cardiovasc Electrophysiol. 2000;11:405-12.

21. Kawaguchi N, Kobayashi Y, Miyauchi Y, Atarashi H, Takano T, Hayakawa H The incidence and clinical significance of junctional rhythm remaining after termination of radiofrequency current in patients with atrioventricular nodal reentrant tachycardia. Jpn Circ J. 1999;63:865-72.

22. Iakobishvili Z, Kusniec J, Shohat-Zabarsky R, Mazur A, Battler A, Strasberg B Junctional rhythm quantity and duration during slow pathway radiofrequency ablation in patients with atrioventricular nodal re-entry supraventricular tachycardia. Europace. 2006;8:588-91.

23. Irons GV, Orgain ES. Digitalis-induced dysrhythmias and their management Prog Cardiovasc Dis. 1996;8:539-69.

24. Wieland JM, Marchlinski FE. Electrocardiographic response of digoxin-toxic fascicular tachycardia to Fab fragments: implication for tachycardia mechanism. PACE. 1986;9:727-38.

25. Betts TR, Ho SY, Sanchez-Quintana D, Roberts PR, Anderson RH, et al. Threedimensional mapping of right atrial activation during sinus rhythm and its relationship to endocardial architecture. J Cardiovasc Electrophysiol. 2002;13:1152-9.

26. Betts TR, Roberts PR, Ho SY, Morgan JM. High density endocardial mapping of shifts in the site of earliest depolarization during sinus rhythm and sinus tachycardia. PACE. 2003;26:874-82.

27. Jones SB, Euler DE, Randall WC, Brynjolfsson G, Hardie EL. Atrial ectopic foci in the canine heart: hierarchy of pacemaker automaticity. Am J Physiol. 1980;7: H788-93.

28. Rigel DF, Lathrop DA. Vasoactive intestinal peptide enhances automaticity of supraventricular pacemakers in anesthetized dogs. Am J Physiol. 1991;261: H463-8.

29. Tan AY, Zhou S, Jung BC, Ogawa M, Chen LS, Fishbein MC, et al. Ectopic atrial arrhythmias arising from canine thoracic veins during in vivo stellate ganglia stimulation. Am J Physiol Heart Circ Physiol. 2008;295:H691-8. 